\title{
SYNTHESIS AND CHARACTERIZATION OF PURE-SILICA- ZEOLITE-BETA MEMBRANE
}

\author{
Yeong Yin Fong and Subhash Bhatia* \\ School of Chemical Engineering, Engineering Campus, \\ Universiti Sains Malaysia, Seri Ampangan, 14300 Nibong Tebal, \\ Seberang Perai Selatan, Pulau Pinang. Malaysia
}

Received 17 June 2005

\begin{abstract}
The semiconductor industry needs low dielectric constant (low k-value) materials to more advance microprocessor and chips by reducing the size of the device features. In fabricate this context, a new material with lower $k$ value than conventional silica ( $k=3.9-4.2$ ) is needed in order to improve the circuit performance. As per the recent International Semiconductor Technology plan, a low-k material with a $\mathrm{k}=1.6$ will be needed by 2010 . The choice of the inorganic zeolite membrane is an attractive option for low $k$ material and suitable for microprocess application.

In the present study, a pure silica zeolite beta membrane coated on the non-porous stainless steel support was synthesized using in situ crystallization of a gel with the composition of $\mathrm{SiO}_{2}: 0.6$ TEAOH : $0.6 \mathrm{HF}: 10.1 \mathrm{H}_{2} \mathrm{O}$. The crystallization was carried in the presence of tetraethylammonium hydroxide $\mathrm{TEA}(\mathrm{OH})$ as structure directing agent, fumed silica, HF and deionized water at $\mathrm{pH}$ value of 9 . The crystallization under hydrothermal conditions at $130^{\circ} \mathrm{C}$ was carried out for the time period of 14 days. The membrane was characterized by X-Ray Diffraction ( XRD ), Thermogravimetric Analysis ( TGA ), Nitrogen Adsorption and Scanning Electron Microscope ( SEM ) .

SEM micrographs show highly crystalline, truncated square bipyramidal morphology of pure silica zeolite beta was coated on the non-porous stainless steel support. The membrane dielectric constant, k-value was measured as 2.64 which makes it suitable for the microprocessor applications.
\end{abstract}

Keywords: Zeolite membrane, zeolite BETA and microprocessor

\section{INTRODUCTION}

There has been a constant research effort in the search for materials with low dielectric constants, which might be used in advanced microprocessor. These materials can reduce the RC delay ( $\mathrm{R}$ for resistance and $\mathrm{C}$ for Capacitance), crosstalk noise, and power dissipation. The microprocessor properties requirements are: thermally stable at temperature $>450^{\circ} \mathrm{C}$, low water absorption, low thermal expansion, low dielectric constant, high mechanical strength, high thermal conductivity, good chemical stability, good electrical properties, good adhesion to a

\footnotetext{
*Corresponding author e-mail: chbhatia@eng.usm.my
} 
variety of surfaces and substrate, low reactivity with conductor metals at elevated temperatures [1 - 3].

The microprocessor manufacturing industry is in a search for dielectrics with a lower $\mathrm{k}$ value than dense silicon dioxide $(\mathrm{k}=3.9-4.2)$. The industry is looking for materials with a $\mathrm{k}$ value lower than 3, and optimally lower than 2.2. These materials will be needed for the design of very small devices, e.g., $100 \mathrm{~nm}$, features. A large variety of polymers have been proposed for use as materials with low dielectric constants for applications in microelectronics. Poly (tetrafluoroethylene) exhibits the lowest dielectric constant with $\mathrm{k}<2$. Polymers are potentially attractive materials for this application. The low thermal conductivity of the polyamide, and moisture uptake were identified to be the key problems for further developments. Dielectric constants below 2.5 will probably not be possible for polymers with thermal stabilities of $450^{\circ} \mathrm{C}$ or even $400^{\circ} \mathrm{C}$ [2]. In addition there is concern that these may react with metals used as conductors at elevated temperatures [3].

Dense (nonporous) pure silica has been the material of choice as dielectrics since beginning of the semiconductor industry. However, an important and promising approach to reduce $\mathrm{k}$ is to introduce porosity into silica. Currently, there are three major classes of porous silica: Sol-gel silica, Surfactant -templated mesoporous silica, Pure silica zeolite. Although sol-gel silica and surfactant -templated mesoporous silica having extremely low k-value ( $k=1.1-1.3$ ), other characteristics like low mechanical strength, low thermal conductivity and hydrophilicity causing both of these materials can not be used as a low dielectric constant materials [ 4 - 11 ].

Zeolites are inorganic microporous crystalline material [12, 13] and have their uniform micropore structure, good thermal stability, high mechanical strength, heat conductivity and resistance to relatively extreme chemical environment due to their dense crystalline backbone drawn much attention in the recent year [14]. Although the aluminosilicate zeolites are hydrophilic, pure silica zeolites are hydrophobic which could help to reduce water adsorption [4]. 'Pure silica zeolites' are silica zeolites having only silicon and virtually no aluminium. Pure silica zeolite beta (PSZ BEA) is a high-silica zeolite possesses a three-dimensional pore structure, consisting of three sets of mutually perpendicular 12-membered ring channels. Currently there are more than 130 zeolites and zeolite type materials available. However, only 13 of them have been synthesized in pure silica form [13]. Although there are many reports on zeolite films, reports on zeolite BEA film are very limited.

The conventional methods for the synthesis of Al-containing zeolite beta using $\mathrm{TEA}^{+}$ions as template in a basic medium have not been successful in the preparation of the pure silica BEA structure, since the aluminum species seem to be required for the nucleation step of the crystallization [15]. In situ crystallization is a preferable method for the synthesis of pure silica zeolite membrane. In this method, a support is immersed in a required synthesis mixture in a Teflon-lined autoclave. Under controlled hydrothermal conditions, a zeolite layer is formed on the surface of the support either by the accumulation of zeolite crystals homogenously nucleated in the synthesis mixture or by the growth of zeolite crystal heterogeneously nucleated on the support surface [16]. The synthesis of pure silica zeolite BEA has been achieved by Corma and coworkers [17] using quaternary ammonium hydroxide usually tetraethylammonium hydroxide (TEAOH) as template and fluoride ion at near neutral $\mathrm{pH}$.

Therefore, thin films for use as dielectric in semiconductor and other devices are prepared from silica zeolites, preferably pure silica zeolites such as pure-silica MFI [4]. The films have low $\mathrm{k}$ values, generally below about 2.7, ranging downwards to $\mathrm{k}$ values below 2.2. The films have relatively uniform pore distribution, good mechanical strength and adhesion, are relatively little affected by moisture, and are thermally stable [ 3,4$]$.

The present work is focused on synthesis and characterization of Pure-Silica-Zeolite Beta (BEA) membrane coated on the non- porous stainless steel support, as low dielectric constant material, suitable for microprocessor applications. 


\section{EXPERIMENTAL}

\subsection{Support cleaning}

A non-porous stainless steel disc support with $25 \mathrm{~mm}$ diameter was first cleaned with the detergent. The stainless steel support was soaked for $30 \mathrm{~min}$ in $1 \mathrm{M} \mathrm{NaOH}$ solution followed by soaking in $1 \mathrm{M} \mathrm{HNO}_{3}$ solution. The support was washed thoroughly with deionized water in the ultrasonic bath for $10 \mathrm{~min}$.

\subsection{Synthesis gel preparation}

The fumed silica (99.9 wt\%, Fluka) is added slowly to an aqueous solution of tetraethylammonium hydroxide TEA(OH) ( $35 \mathrm{wt} \%$ ) in a polypropylene bottle under stirring at room temperature, $25^{\circ} \mathrm{C}$. The stirring was continued for a period of nearly 3 hours until a semitransparent mixture was obtained. Aqueous HF (40 wt\%) was added dropwise under vigorous stirring. A homogenous, high viscous solid like gel was formed. The final gel molar composition obtained was $\mathrm{SiO}_{2}$ : $0.6 \mathrm{TEAOH}: 0.6 \mathrm{HF}: 10.1 \mathrm{H}_{2} \mathrm{O}$.

\subsection{In situ crystallization}

The gel was transferred to a Teflon-lined Parr reactor (Model 4842) for crystallization under hydrothermal conditions The crystallization temperature was kept constant at $130^{\circ} \mathrm{C}$ and time for crystallization was nearly 14 days. To obtain pure silica zeolite BEA films, non-stainless steel support was placed vertically inside the reactor and completely covered by the gel during crystallization. The synthesized membrane and solid product were washed thoroughly with deionized water in the ultrasonic bath. The solid product was then separated from the remaining liquid phase by filtration. After that, the sample was dried for $8 \mathrm{~h}$ at $100^{\circ} \mathrm{C}$ in the oven before calcined in static air at $500^{\circ} \mathrm{C}$ for $10 \mathrm{~h}$ with ramping rate $5^{\circ} \mathrm{C} / \mathrm{min}$ to remove the TEAOH template from the zeolite framework.

\subsection{Characterization}

The samples were characterized using X-ray diffraction (XRD), (SEM) scanning electron microscope, thermogravimetric analysis (TGA) and nitrogen gas adsorption-desorption isotherm. XRD analysis was done using Siemen diffractometer (model 1820) with graphite monochromated $C u K_{\alpha}(\lambda=1.5406 \AA)$ source operated at $40 \mathrm{kV}$ and $40 \mathrm{~mA}$. The SEM of the membrane was studied by using SUPRA 50VP-23-57 scanning electron microscope (SEM), operate at $3.00 \mathrm{kV}$. Nitrogen gas adsorption-desorption measurement was performed at $77 \mathrm{~K}$ on a Autosorb I (Quantachrome Automated Gas Sorption System). The samples were first degassed for $3 \mathrm{~h}$ under vacuum at $300^{\circ} \mathrm{C}$ prior to the analysis. TGA of as-synthesized and calcined pure silica zeolite was conducted at $700^{\circ} \mathrm{C}$ by using Perkin Elmer TGA 7, at a heating of $10^{\circ} \mathrm{C} / \mathrm{min}$ under a nitrogen flow rate of $30 \mathrm{ml} / \mathrm{min}$. For dielectric measurement, aluminium dots with a dimension $3 \mathrm{~mm} \times 3 \mathrm{~mm}$ were deposited on the film by thermal evaporation with pressure $3.2 \times$ $10^{-5}$ bar and low temperature (4.5 A, LT) through a contact mask, using vacuum coating unit (Edwards Auto 306). The capacitance of metal-membrane-metal structure was measured at a frequency of $100 \mathrm{kHz}$ on capacitance voltage system with standard Signaton microprobe station and micropositioner. The capacitance of the membrane was measured in a voltage range of $-4 \mathrm{~V}$ to $4 \mathrm{~V}$. The k-value of the membrane was calculated using equation (1):

$$
\varepsilon_{r}=\frac{C t}{\varepsilon_{o} A}
$$


where $\varepsilon_{r}$ is dielectric constant, k-value of the membrane, C is capacitance of the membrane, Faraday (F), $\varepsilon_{o}$ is constant, $8.85 \times 10^{-12}$ Faraday $/ \mathrm{m}$, A is cross sectional area aluminium dot, $\mathrm{m}^{2}$ and $\mathrm{t}$ is thickness of the membrane, $\mathrm{m}$.

\section{RESULTS AND DISCUSSION}

X-ray diffraction (XRD) technique was used to identify component phases and to obtain relative crystallinity of the synthesized membrane. Figure 1 illustrates typical X-ray diffraction pattern of the synthesized membrane. The main peak appearing at $2 \theta=22.5^{\circ}$, with an interplanar dspacing $\mathrm{d}_{\mathrm{hkl}}=3.912$, is a typical of the zeolite BEA topology and thus showing that the zeolite BEA membrane was formed. The high crystallinity of zeolite BEA membrane can be observed from the XRD pattern.

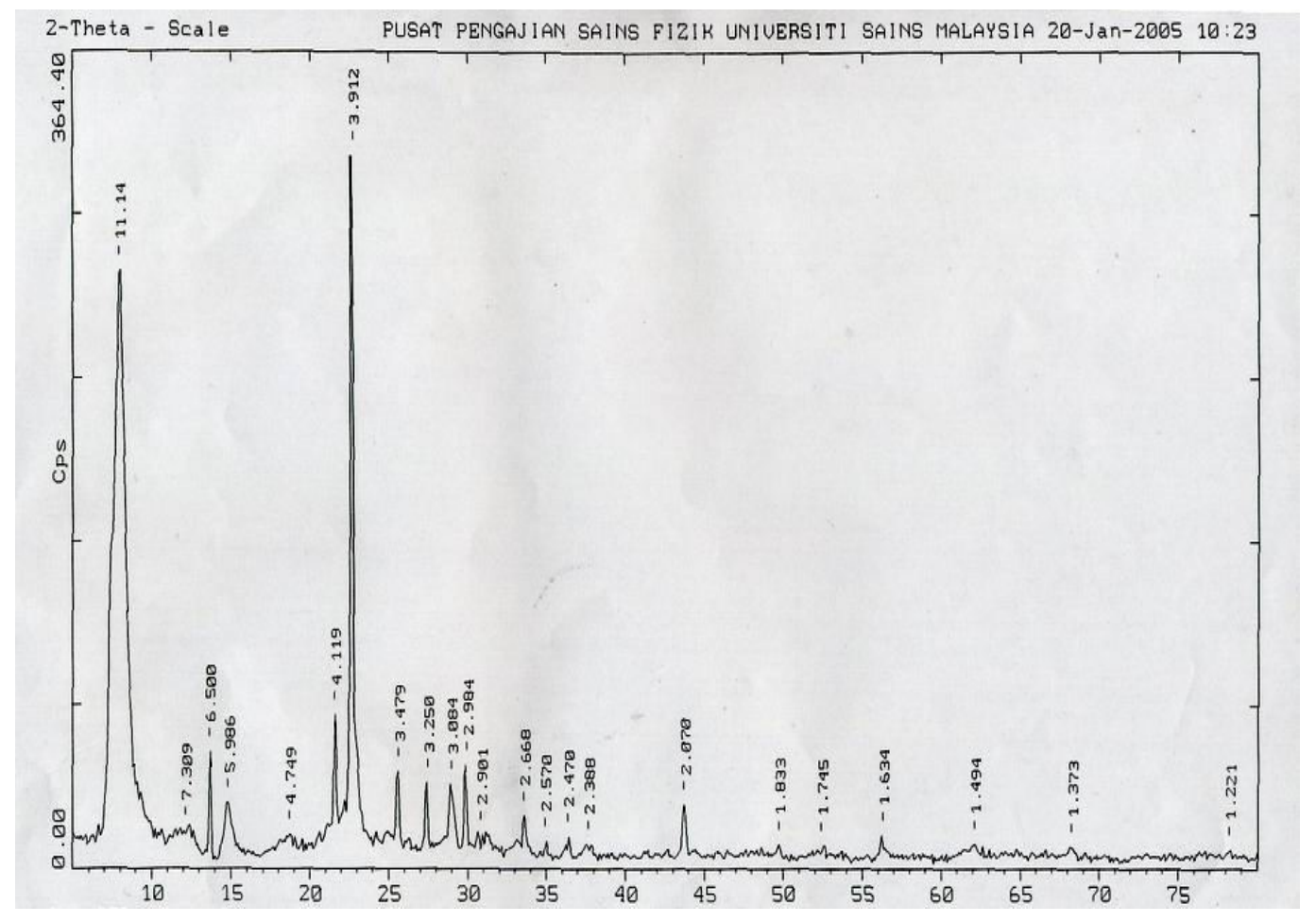

Fig. 1: XRD pattern for pure silica zeolite BEA membrane growth on the support

The membrane grown on the non-porous steel support was also examined by SEM. SEM micrographs of the membrane reveal a well intergrown, continuous and polycrystalline film as shown in Fig. 2(a). There were no obvious defects such as intercrystal gaps and cracks present in the membrane. Figure 2(b) shows SEM micrographs of highly crystalline, truncated square bipyramidal morphology pure silica zeolite BEA coated on stainless steel after calcination at the temperature of $500^{\circ} \mathrm{C}$ for 10 hours. The crystal size of $\sim 2.5 \mu \mathrm{m}$ fully covered the surface of the support had a membrane thickness of around $2 \mu \mathrm{m}$ ( Fig. 2(c) ). 


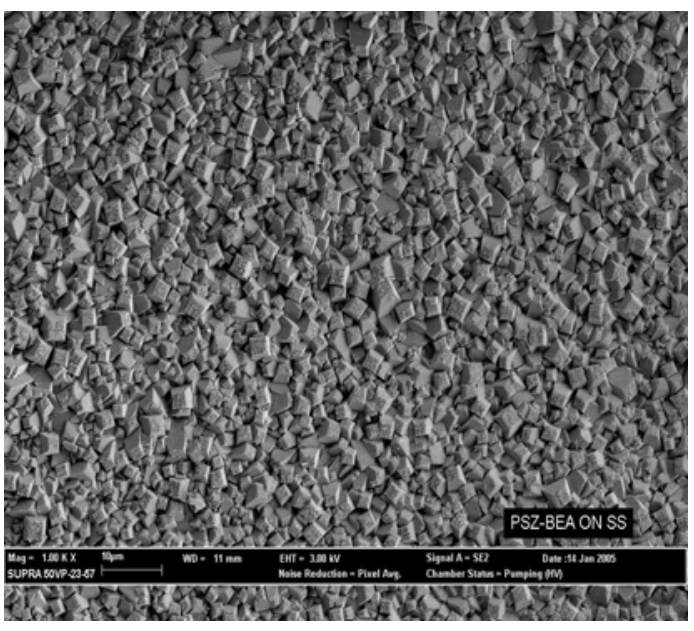

(a)

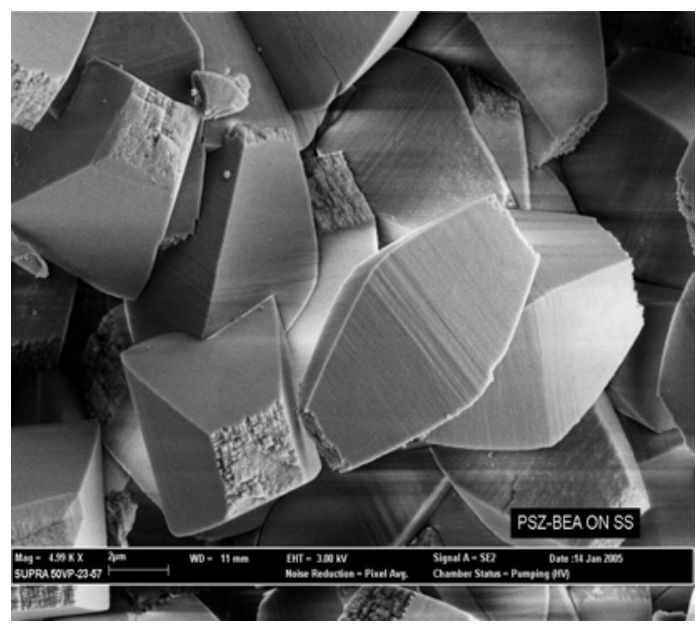

(b)

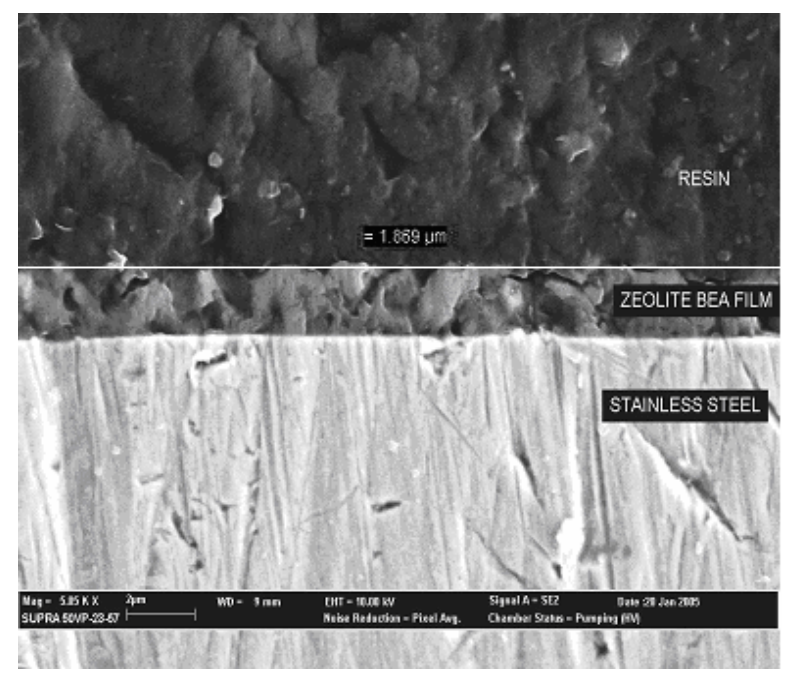

(c)

Fig. 2: SEM micrographs of pure silica zeolite BEA coated on non porous stainless steel support after calcinations. (a), (b): Top view (without polishing); (c): Cross sectional view of SEM micrographs of pure silica zeolite BEA coated on non porous stainless steel support after calcinations (after polishing)

TGA provided valuable information on the hydrophilicity/hydrophobicity of the sample and thermal decomposition of the structure directing agent (SDA). The as-synthesized sample (powder form) was dried at $100^{\circ} \mathrm{C}$ overnight and was used for the study of thermal decomposition of SDA. The TGA curve for as-synthesized pure sample is shown in Fig. 3(a). The calcined sample was used to measure the moisture adsorption and the TGA curve for calcined sample is shown in Fig. 3(b).

Figure 3(a) shows that, the weight \% loss of as-synthesized pure silica zeolite BEA sample was constant when the temperature reached $500^{\circ} \mathrm{C}$. The weight $\%$ loss was $22.7 \%$ which resulted due to the loss of the SDA content of the sample (22.7 wt \%) of the sample.

The weight \% loss of the calcined sample exhibited a very low moisture content of $1.15 \%$ as shown in Fig. 3(b). The moisture could be readily removed at a temperature $200^{\circ} \mathrm{C}$ and the 
weight \% loss was constant for temperature the above $200^{\circ} \mathrm{C}$. This shows that the pure-silicazeolite BEA is a hydrophobic membrane with a high thermal strength. The moisture content has significant influence on the $\mathrm{k}$ value of the membrane, thus the lower moisture content of the sample can result in a lower $\mathrm{k}$ value.

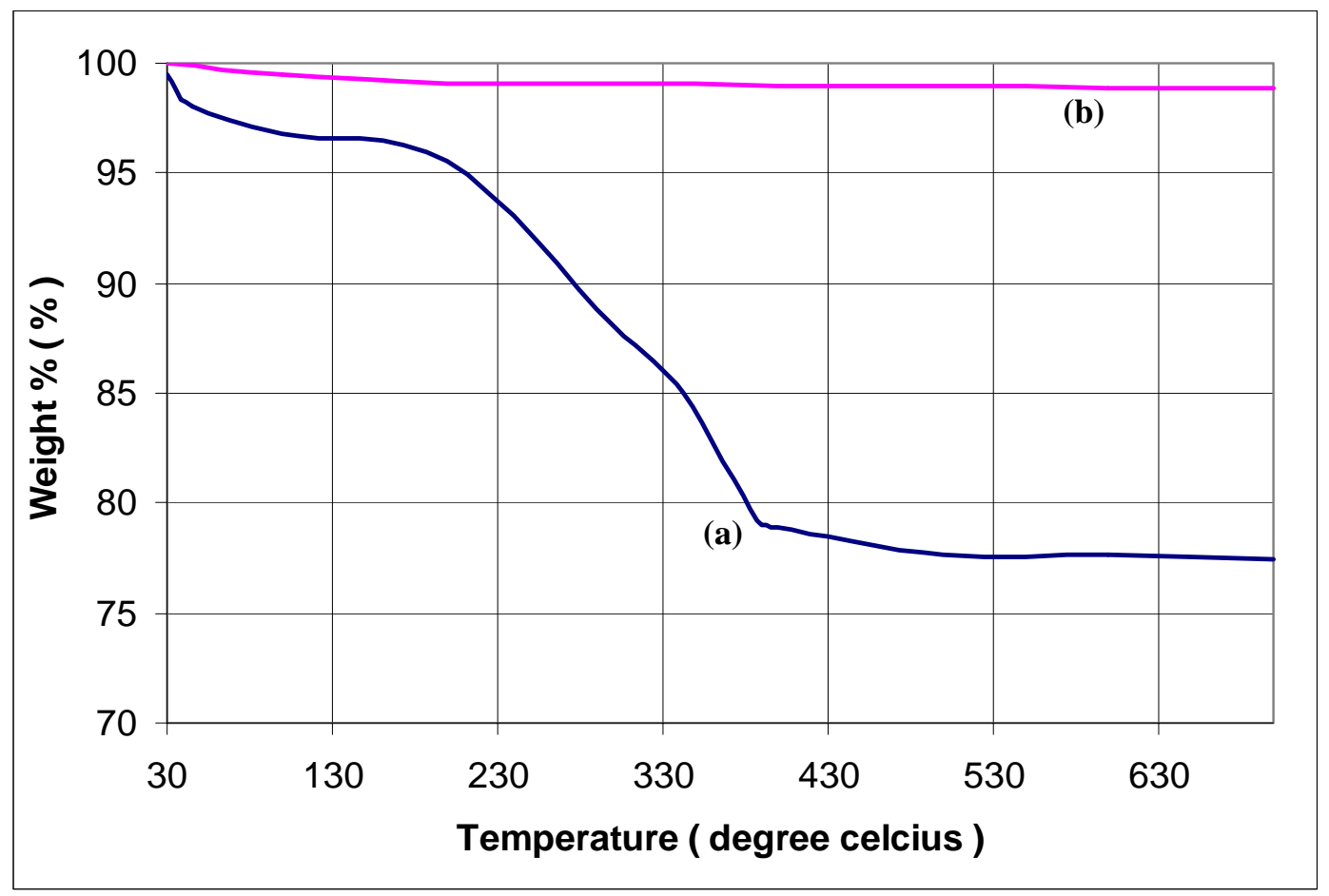

Fig. 3: Thermogravimetric Analysis ( TGA ) curve for (a): As- synthesized; (b): Calcined, pure silica zeolite BEA

The BET surface area and pore volume of the calcined samples were measured by nitrogen adsorption-desorption isotherm. The BET surface area and average pore diameter for the samples are presented in the Table 1. Pure silica zeolite BEA was a microporous solid exhibiting a high proportion of microporosity (90\%). The total pore volume obtained in this experiment was $0.29 \mathrm{~cm}^{3} / \mathrm{g}$ closed to the value $0.26 \mathrm{~cm}^{3} / \mathrm{g}$ reported in the literature. The $\mathrm{t}$ method micro pore volume measured was $0.21 \mathrm{~cm}^{3} / \mathrm{g}$, which was also closed to the t-method micro pore volume, $0.23 \mathrm{~cm}^{3} / \mathrm{g}$ as reported in literature for pure silica zeolite BEA [16].

Table 1: Surface area and average pore size of the calcined sample

\begin{tabular}{lc}
\hline \multicolumn{1}{c}{ Data } & Pure silica zeolite BEA obtained in the present work \\
\hline BET surface area $\left(\mathrm{m}^{2} / \mathrm{g}\right)$ & 435 \\
Total pore volume $\left(\mathrm{cm}^{3} / \mathrm{g}\right)$ & 0.29 \\
Average pore diameter $(\AA)$ & 6.7 \\
\hline
\end{tabular}

A smooth curve without any sharp increase in nitrogen adsorption-desorption isotherm for the samples was obtained and presented in Fig. 4. The volume adsorbed varied from $142 \mathrm{~cm}^{3} / \mathrm{g}$ to $190 \mathrm{~cm}^{3} / \mathrm{g}$ for P/Po reading from 0.1 to 1.0 . The isotherm curves reported in the literature for pure silica crystalline zeolite BEA, and the volume varried from $150 \mathrm{~cm}^{3} / \mathrm{g}$ to $185 \mathrm{~cm}^{3} / \mathrm{g}$ for 290 
$\mathrm{P} /$ Po reading from 0.1 to 1.0 [16]. In case of partial crystalline pure silica zeolite BEA, there was a jump in the isotherm curve for P/Po reading from 0.9 to 1.0 as reported in the literature. The volume varried was from $90 \mathrm{~cm}^{3} / \mathrm{g}$ to $270 \mathrm{~cm}^{3} / \mathrm{g}$ for P/Po reading from 0.1 to 1.0 [16]. Thus, we can conclude that the pure silica zeolite BEA synthesized was highly crystalline in the present study. The high crystallinity of the sample is also confirmed from the XRD pattern.

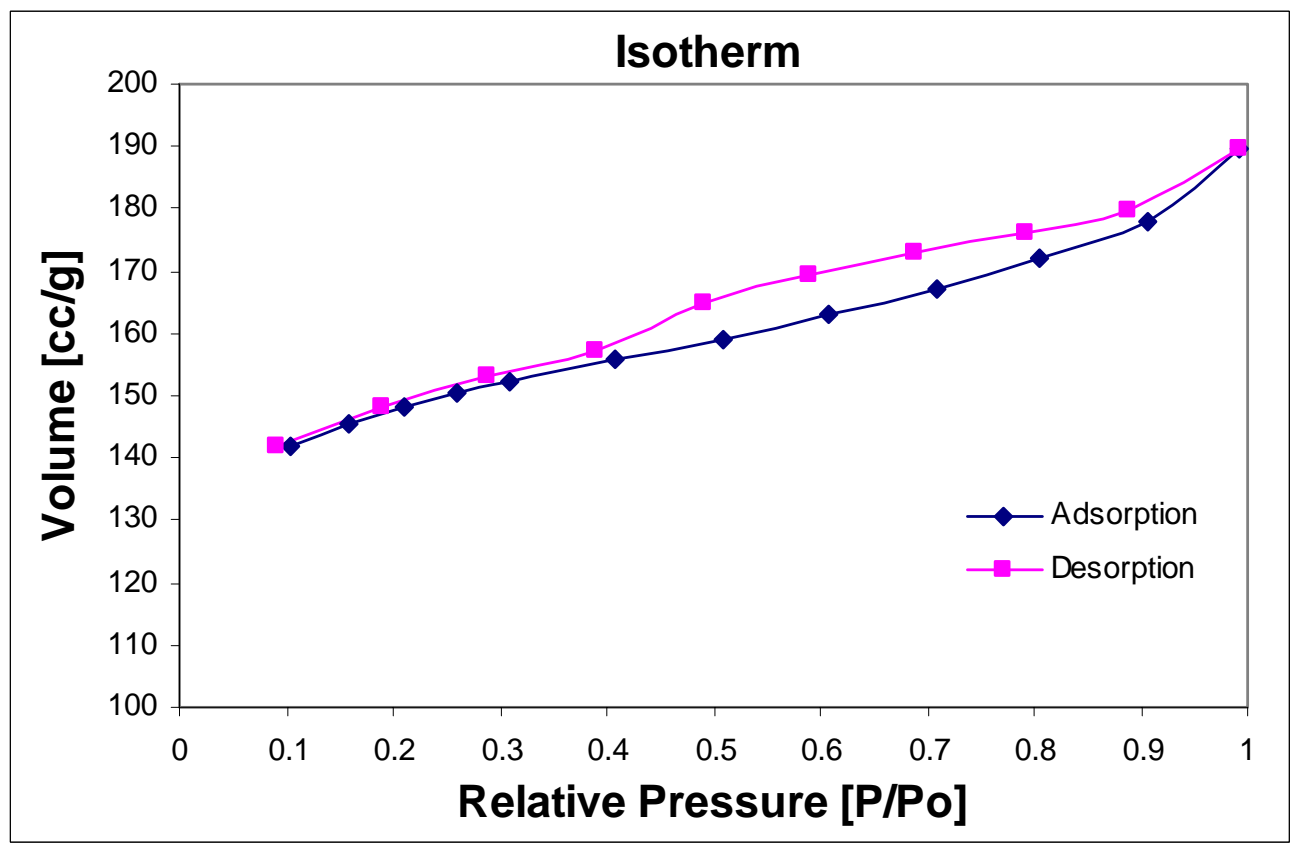

Fig. 4: Nitrogen adsorption-desorption isotherms for the samples

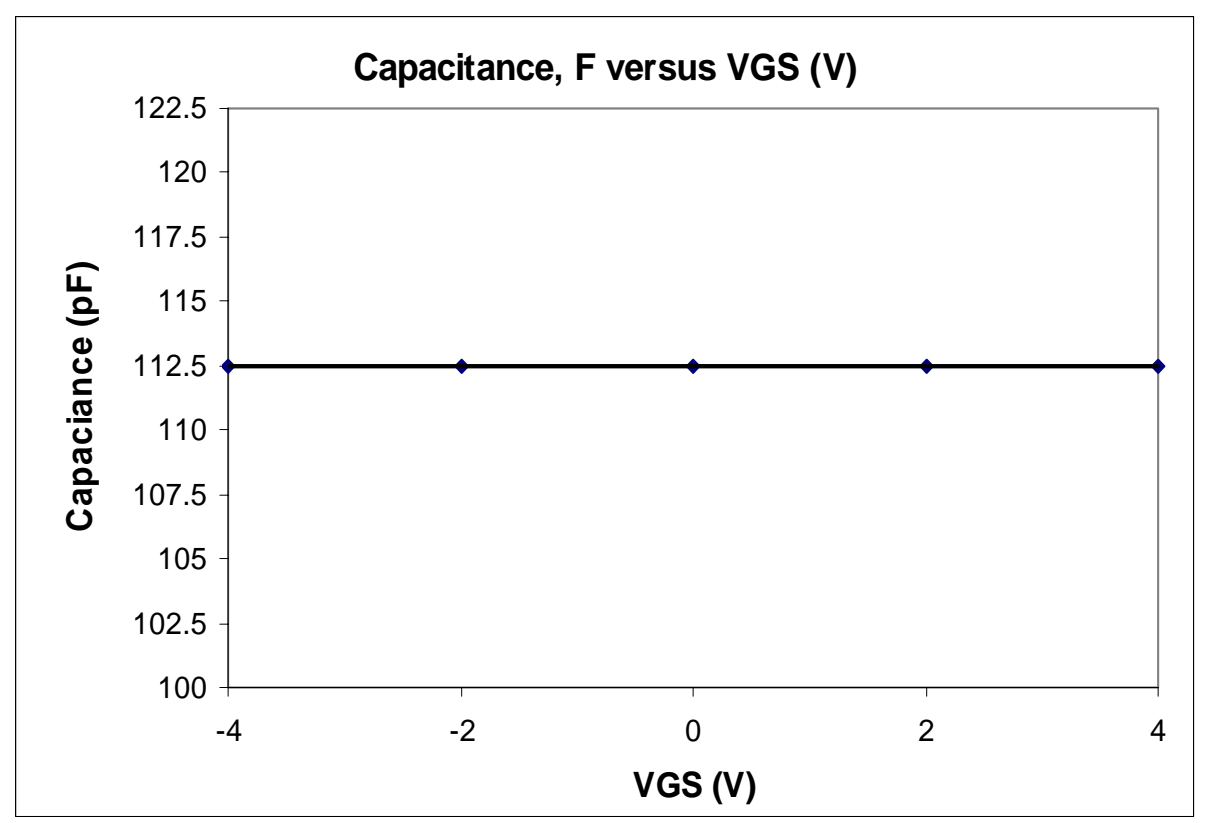

Fig. 5: Capacitance of calcined pure silica zeolite BEA membrane on stainless steel support (thickness: $1.869 \mu \mathrm{m}$ and the measurement area: $9 \times 10^{-6} \mathrm{~m}$ ) 
The thickness of the pure silica zeolite BEA membrane coated on the non-porous stainless steel support is required for the calculation of k-value. To measure the thickness of the membrane, the sample was moulded using epoxy. The sample was then polished with sand papers using grit number of 180, 240, 320, 600 and 2000, respectively. It was followed by fine polishing with diamond suspension from 45 micron, 30 micron, 15 micron to 9 micron using a Grinder polisher. The polishing did not cause cracking of the membrane. The membrane strongly adhered to the substrate even after polishing. This shows that the pure silica zeolite BEA membrane had outstanding mechanical strength and adhesion with the support.

Figure 5 shows the capacitance of the membrane as a function of voltage. The capacitance value of the membrane, with the thickness of $1.869 \mu \mathrm{m}$ and the measurement area of $9 \times 10^{-6} \mathrm{~m}$, was constant in the range of $-4 \mathrm{~V}$ to $4 \mathrm{~V}$ as $112.5 \times 10^{-12} \mathrm{~F}$. These values were substituted into the equation (1) and found that the dielectric constant, k-value studied in the present work was 2.64, which was lower than $\mathrm{k}$-value as 3 . Thus, pure silica zeolite membrane is a promising low-k material and suitable for microprocessor application

\section{CONCLUSIONS}

Pure silica zeolite BEA membrane coated on the non-porous stainless steel support was synthesized. The sample was characterized by x-ray diffraction (XRD), scanning electron microscope (SEM), thermogravimetric analysis (TGA) and nitrogen adsorption-desorption isotherm.

XRD result shows that the pure silica zeolite BEA membrane was successfully formed, under hydrothermal crystallization at $130^{\circ} \mathrm{C}$ within a period of 14 days. The thermogravimetric analysis results show that pure silica zeolite beta is highly hydrophobic membrane with very low moisture content of $1.15 \%$. The SEM results revealed that a well intergrown, continuous and polycrystalline membrane was coated on the support. The structural defects like intercrystal gaps and cracks were found to be absent.

The membrane was strongly adhered to the support even after polishing. The membrane had outstanding mechanical strength and adhesion with the support. The membrane dielectric constant k-value was 2.64 which makes it suitable for the microprocessor applications.

\section{ACKNOWLEDGEMENT}

The financial support provided by Universiti Sains Malaysia under Fundamental Research Grant Scheme (FGRS) (Account No : 6070013) is duly acknowledged.

\section{REFERENCES}

1. Treichel, H., Ruhl, G., Ansmann, P., Wijrl, R.., Muller, Ch., and Dietlmeier, M. (1998), Microelectric Engineering., vol. 40, pp. 1-19.

2. Maier, G. (2001), Progress in Polymer Science, vol. 26, pp. 3-65.

3. Yan, Y., Wang, Z., Wang, H., and Zheng, B. (2003), U.S. Patent 6,573,131.

4. Mitra, A., Cao, T., Wang, H., Wang, Z., Huang, L., Li, S., Li, Z., and Yan, Y. (2004), Ind. Eng. Chem. Res., vol. 43, pp. 2946-2949.

5. Hrubesh, L.W., Keene, L.E., and LaTorre V.R. (1993), Journal of Material Research, vol. 8, pp. 1736-1741.

6. Hrubesh, L.W. (1998), Journal of Non-Crystalline Solids, vol. 225, pp. 335-342. 
7. Seraji, S., Wu, Y., Forbess, M., Limmer, S.J., Chou, T., and Cao, G.Z. (2000), Advanced Materials, vol. 12, pp. 1695-1698.

8. Zhao, D., Yang, P., Melosh, N., Feng, J., Chmelka, B.F., and Stucky, G.D. (1998), Advanced Materials, vol. 10, pp. 1380-1385.

9. Baskaran, S., Liu, J., Domansky, K., Kohler, N., Li, X.H., Coyle, C., Fryxell, G.E., Thevuthasan, S., and Williford, R.E. (2000), Advanced Materials. vol. 12, pp. 291-294.

10. Davis, M.E. (2002), Nature. vol. 417, pp. 813-821.

11. Yu, S.Z., Wong, T.K.S., Pita, K., and Hu, X. (2002), Journal of Vacuum Science and Technology; B. vol. 20, pp. 2036-2042.

12. Breck, DW. (1974), Zeolite Molecular Sieves Structure, Chemistry, and Use. New York: Wiley.

13. Baerlocher, C., Meier, W.M., and Olson, D.H. (2001), Atlas of Zeolite Framework Types. Amsterdam: Elsevier.

14. Shan, W., Zhang, Y., Yang, W., Chen, K., Zi, G., Ye, Y., and Tang, Y. (2004), Microporous Mesoporous Materials. vol. 69, pp. 35-42.

15. David, P.S., Rafael, V.G., Sanchez, P., Raul, S., and Rodriguez, L. (2001), Microporous Mesoporous Material. vol. 46, pp. 35-46.

16. Gang, L., Eiichi Kikuchi, and Masahiko, M. (2003), Microporous Mesoporous Material. vol. 62, pp. 211-220.

17. Rafael, V.G. and David, P.S. (2001), Journal of Material Chemistry. vol. 11, pp. 2391-2407. 